\title{
Beziehungen sichtbar machen - Debatten erweitern. Reproduktionstechnologien denken mit Marilyn Strathern
}

Die britische Sozial- und Kulturanthropologin Marilyn Strathern hat die Entwicklung, Einführung und gesellschaftliche Verbreitung der Technologien assistierter Reproduktion von Anfang an kritisch begleitet. Seit 30 Jahren reflektiert sie deren Ausformungen und viele ihrer Schülerinnen stehen heute im Zentrum eines expandierenden Forschungsfelds zum Thema Verwandtschaft im Zeitalter der Reproduktionstechnologien (z. B. Franklin 2002; 2013; Edwards 2000; Konrad 2005; Thompson 2007). Sie alle positionieren sich an der Schnittstelle von Sozialanthropologie, STS (Social Studies of Science and Technology) und Gender Studies. In Deutschland sind Marilyn Stratherns komplexe und eigenwillige Schriften außerhalb der Sozial- und Kulturanthropologie bislang jedoch nur wenig bekannt und keine ihrer Monographien und kaum einer ihrer Texte ist ins Deutsche übersetzt. Mit der hier vorgelegten deutschsprachigen Erstveröffentlichung des Aufsatzes "Tauschverhältnisse und Konsumverhältnisse. Der Embryo als zukünftiger Konsument« aus dem Jahr 1990 wollen wir beginnen, diese Lücke zu schließen und eine Möglichkeit dafür schaffen, das Denken von Marilyn Strathern in die hiesige feministische Forschung zum Thema Reproduktionstechnologien miteinzubeziehen. Warum wir das für ein relevantes und gewinnbringendes Unterfangen halten, legen wir in diesem Beitrag dar. Zugleich führt unser Aufsatz ein in die thematischen Kontexte und methodologischen und analytischen Strategien von Marilyn Strathern. Das Theorie-Interesse dieser in der britischen Sozialanthropologie sozialisierten Forscherin ist doppelt geschärft, durch feministische Forschung zum einen und papua-neuguineische Ethnographien zum zweiten. Entsprechend ist auch ihr Blick auf Gegenwartsphänomene geprägt durch ihre ethnographischen Arbeiten im Hochland von Papua-Neuguinea in der Region um Mount Hagen (Strathern 1988) und durch eine fortgesetzte Bezugnahme auf die Historizität und Vielfalt der eigenen, westlichen Kultur (z.B. Strathern 1981, eine Ethnographie zu 
Verwandtschaft in einem Dorf in Essex). Im Nachdenken über Veränderungsprozesse in westlichen/euroamerikanischen/globalisierenden Gegenwartsgesellschaften mobilisiert Strathern Kategorien und Konzepte aus der Gegend von Mount Hagen und benutzt diese für Analogiebildungen und relationale Bezugnahmen. Das gegenseitige Beleuchten und Durcharbeiten von europäischen und melanesischen Vorstellungsweisen und Begriffen bieten ihr Denkmöglichkeiten, um den für westliche Wissenschaften und Alltagswelten selbstverständlichen Formen des Nachdenken über die eigene Kultur und Gesellschaft auf die Spur zu kommen. Wie sie damit das Thema Reproduktion - Verwandtschaft - Geschlecht aufschließt, soll im Folgenden veranschaulicht werden.

\section{Text und Kontext}

Der übersetzte Text wurde 1990 als Vortrag mit dem Titel "Partners and Consumers. Making Relations Visible« auf der Konferenz »The Gift and it's Transformation" am National Humanities Center North Carolina gehalten und anschließend mehrfach veröffentlicht: 1991 in der Zeitschrift New Literary History (Strathern 1991), 1992 in dem Sammelband »Reproducing the Future«, in dem verschiedene Aufsätze der Autorin zum Thema Reproduktionstechnologie und zum Thema Verwandtschaft in Melanesien zusammen gestellt sind (Strathern 1992) sowie 1997 als Beitrag in dem von Alan Schrift edierten Buch "The Logic of the Gift" (Strathern 1997). ${ }^{1}$

Der Text ist geschrieben am Ende der Thatcher-Ära mit all ihren sozialen und kulturellen Verwerfungen und neoliberalen Ausrichtungen ${ }^{2}$, zu einem Zeitpunkt, an dem die ersten Erfolge der künstlichen Befruchtung schon ein Jahrzehnt zurück lagen und nun rechtliche Regulierungen erforderlich machten. Louise Brown, das erste mit IVF-Technologie gezeugte Kind, kam 1978 in Oldham bei Manchester auf die Welt und 1990 verabschiedete das britische Parlament den ersten "Human Fertilisation and Embryology Act«, dem umfängliche öffentliche Debatten voraus

1 Grundlage der Übersetzung wie auch unserer Ausführungen ist Strathern 1992.

2 Die kulturelle Bedeutung der von Thatcher vollzogenen politischen und ökonomischen Modernisierung zeigt sich nicht zuletzt in der häufig zitierten Interviewpassage, in der Thatcher (zivil)gesellschaftliche Institutionen und Reglements als obsolet darstellte und erklärte: "there's no such thing as society. There are individual men and women and there are families. And no government can do anything except through people, and people must look after themselves first. It is our duty to look after ourselves and then, also, to look after our neighbours" (Thatcher 1987, 10). 
gegangen waren. In Deutschland erblickte Oliver Wimmelbacher als weltweit siebtes mit Hilfe von In-Vitro-Fertilisation gezeugtes Kind 1982 in der Uni-Klinik Erlangen-Nürnberg das Licht der Welt und Ende 1990 passierte das »Embryonenschutzgesetz« den Bundestag (Klotz 2014). Anlass und Gegenstand der Gesetzgebung und der sie begleitenden Diskussionen war, wie Strathern herausstellt, eine historisch neue Entität: der durch Reproduktionstechniken erzeugte menschliche Embryo in seinem frühen Stadium, lebendig, aber außerhalb des mütterlichen Körpers, eine durch bildgebende Verfahren sichtbar gemachte und durch In-Vitro-Technologien hergestellte totipotente Zellstruktur als "free standing entity" (Strathern 1992a, 4).

Während in England die öffentliche Debatte von Lebensschützern initiiert, von der Warnock-Kommission durch Expert_innen vorbereitet und schließlich im Parlament auf das Argument der Gewährleistung wissenschaftlichen und technischen Fortschritts zum Wohle der Gesellschaft fokussiert wurde (Franklin 1993), stand in der Bundesrepublik in den die parlamentarische Entscheidung begleitenden Experten-Kommissionen die ethische Frage im Mittelpunkt, ob dem Embryo in vitro wie auch dem Embryo im Mutterleib von Beginn an der gleiche Umfang und das gleiche Ausmaß an Schutz wie dem geborenen Menschen zu gewähren sei (Günter/Taupitz / Kaiser 2008). Marilyn Strathern versteht diese Debatten um den rechtlichen Status des Embryos zunächst als Hinweis darauf, dass als selbstverständlich geltende kulturelle Annahmen und Formen des Themas Fortpflanzung und Zeugung durch die Reproduktionstechnologien neu adressiert werden. Die sich verbreitende, normalisierte Nutzung von Technologien der assistierten Reproduktion bewirke, dass grundlegende, weitgehend unhinterfragte kulturelle Verhältnisse und Formen der Gestaltung von Familie und Verwandtschaft in den Blick geraten, z. B. Praktiken und Konzepte wie 'Sex haben`, `Gene weitergeben`, `Gebären`. Vorstellungen, die lange Zeit fraglos als kulturell fest verankerte Grundlagen von Beziehungen zwischen Paaren, Geschwistern, Eltern und Kindern dienten, werden nun als hergestellte und veränderbare Ideen und Praktiken explizit und zum Gegenstand gesellschaftlicher Debatten (Strathern 1992a, 5). Verbunden damit sei auch, in eine Auseinandersetzung über die "Reproduktion von Zukunft" (so der Titel des Sammelbands von 1992) in mehrfachem Sinne einzutreten. In den gesellschaftlichen Bemühungen, "dem Embryo" einen Rechtsstatus zuzuschreiben, zeige sich nicht nur ein Ringen um eine die Zukunft der Technologie gewährleistende Gesetzgebung, sondern werde auch die Frage aufgeworfen, wie die modernen Reproduktionstechnologien die Interaktionen, Vorstellungen und Praktiken 
zwischen Verwandten gestalten und wie sie die Konstitution verwandtschaftlicher Verhältnisse überhaupt betreffen.

Dementsprechend ist Stratherns Interesse an den Reproduktionstechnologien durchweg mit der Frage verbunden, welche Aufforderungen und Möglichkeiten die Techniken bereitstellen, über Verwandtschaft und Verwandtschaftsnetzwerke neu nachzudenken. Statt einen Kulturwandel durch Technik zu postulieren oder eine technische Ersetzung von Körpernatur und geschlechtlichen Körperprozessen zu vermuten, rückt sie die Prozesse des kulturellen (Be-)Deutens in den Blick und zeigt, wie wir das technologisch Neue auf der Folie bereits bestehender Konzepte und Vorstellungen denken. Sie bringt dominante und dissonante Stimmen in den Debatten um Reproduktionstechnologien zu Gehör, macht die hegemonialen Kategorien in diesen Debatten kenntlich, indem sie gleichzeitig alternative Möglichkeiten ins Bild rückt und erweitert diese dann durch scheinbar ähnliche, aber doch ganz verschiedene Kategorien (der Person, der Gabe/Spende, der Relation) aus der ethnographischen Dokumentation zu Papua-Neuguinea. So legt sie eine Grundlage dafür, dass diese anderen Stimmen zumindest potentiell die gegenwärtigen Diskussionen beeinflussen und vielleicht sogar umgestalten können (Hirsch 2014, 41).

\section{Komposition und Denkstil ${ }^{3}$}

Strathern arbeitet durchweg so, dass sie ihre forscherisch-denkende Aktivität situiert, deren kulturelle, soziale und gesellschaftliche Bedingungen und Kontexte sichtbar macht und sie in ihre Reflexionen einbezieht. In dem hier vorliegenden Text bilden drei Aspekte das grundlagentheoretische Fundament ihrer Ausführungen:

1. die gesellschaftliche Verankerung der neuen Reproduktionstechnologien in der westlichen kapitalistischen Warenökonomie,

2. das Spannungsverhältnis von bereits existierenden, ralten kulturellen Formen und dem neuen Wissen über technisch gestützte Formen biologischer und sozialer Reproduktion sowie

3. die Überzeugung, dass die Konzepte, mit denen wir gesellschaftliche Entwicklung fassen, differenziert und erweitern werden müssen.

3 Wir spielen hier nicht auf das Flecksche Theorem an. Es geht uns vielmehr im umgangssprachlichen Sinne um die Typisierung der Art und Weise von Stratherns Schreiben, Denken und Arbeiten. 
Zum Ersten verortet Strathern ihr Nachdenken über die kulturellen und gesellschaftlichen Bedeutungen der Reproduktionstechnologien im gesellschaftlichen Kontext der Modernisierungspolitik Margret Thatchers, die Individualismus und Konsumkultur stärkte, intensivierte und ein Denken beförderte, das die Wahlentscheidung der Einzelperson zum gesellschaftlichen Maßstab für Freiheit erhebt. Strathern sieht diese angebliche Freiheit der Wahl jedoch als gesellschaftlichen Wahl-Zwang, der eng mit einem normativen Imperativ verbunden ist, demzufolge die Subjekte ihre eigene Individualität in seriellen Konsum-Entscheidungen permanent aktualisieren und absichern müssen. In dieser Kultur sind die Repro-Techniken entwickelt worden und in dieser Kultur breiten sie sich aus. Die "Konsumkultur» habe, so Stratherns Überzeugung, die Beschaffenheit der Beziehung zwischen Individuum und Gesellschaft, zwischen Natur und Kultur und die Repräsentationen dieser Beziehungen nachhaltig verändert und das Denken und Handeln der Einzelnen verunsichert (Strathern 1981; 1992a, 12). Die kulturell-gesellschaftliche Verankerung der Neuen Reproduktionstechnologien im Waren- und Konsum-Kapitalismus geht auch - und das ist ein Ausgangspunkt des vorliegenden Textes - in die begrifflich-konzeptionelle Erfassung und Beschreibung reproduktionstechnologischer Aktivitäten ein. So hat sich etwa die Redeweise durchgesetzt, dass Samen und Eizellen gespendet und Gebärmütter geliehen werden. Es gelte, sagt Strathern, diese Tauschverhältnisse genauer zu verstehen: Welche Vorstellungen und Bedeutungen von Geben und Nehmen, von Tausch und Verbundenheit, von Person und Beziehung werden in diesen Bezeichnungen transportiert?

Die zweite Prämisse des Strathernschen Denkens ist epistemologischer Art. Sie stellt heraus, dass die kulturelle Deutung, die gesellschaftliche Aneignung der Technologien der assistierten Reproduktion nur mit Hilfe bereits vorhandener Vorstellungen und Bedeutungen erfolgt, also mit Ideen, die schon Teil des kulturellen Repertoires sind. Als kulturelle Fakten beeinflussen und prägen die vorhandenen Ideen die Repräsentationen, Beschreibungen und Analysen gegenwärtiger wie auch zukünftiger Verwandtschaft (Strathern 1992a, 3). Gleichermaßen sind auch die Reproduktionstechnologien durch bestehende kulturelle Ideen hervorgebracht und werden durch diese handhabbar gemacht und so untersucht Strathern, auf welche der vorhandenen Metaphern, Konzepte und Bilder zur Beschreibung und Analyse der mit den Reproduktionstechniken entstehenden neuen Verhältnisse und Interaktionen Bezug genommen wird und welche anderen Möglichkeiten der Bezugnahme es eventuell geben könnte. 
Drittens ist die Einordnung, Überprüfung und Überarbeitung gängiger Konzepte und Modelle ein organisierendes Prinzip des Strathernschen Schreibens. Im vorliegenden Text kreisen ihre Überlegungen um das Konzept der >Person` und das der >Gabe<; ein thematischer Fokus, der sachlich durch die zu Beginn der 1990er Jahre geführten parlamentarischen Debatten zur rechtlichen Regelung des Umgangs mit dem Embryo und mit dessen Personen-Status begründet ist und gleichzeitig auf Fragen der rechtlichen Regulation von IVF-Verfahren, die auf Eizell- und Samen»spende» aufbauen. Stratherns spezifische Art der Auseinandersetzung mit diesen empirischen Ereignissen besteht darin, den Gegenstand Embryo/Person auf die ihm zugrunde liegenden Konzepte, Prinzipien und Denklogiken hin zu befragen. Konkret interessiert sie, was es bedeutet, den Embryo als >Person $\mathrm{zu}$ entwerfen und welche Mechanismen von Personalisierung oder anders gesagt - welche kulturellen Praktiken des Person-Machens dabei zur Anwendung kommen. Zugleich ist es zentrales Anliegen ihrer Denkarbeit, nach weiteren, möglichen anderen Konzepten und Denkweisen zu suchen und diese in ihre Erwägungen miteinzubeziehen.

Stratherns Überzeugung, dass es bedeutsam sei, mit welchen Konzepten und Begriffen man andere, neue Ideen zu denken versucht (Strathern 1992a, 10), bestimmt auch ihre Art zu schreiben. Sie perspektiviert das Thema Reproduktion vielfältig, indem sie verschiedene Stimmen und Sichtweisen aufruft: Ein Experimentalembryologe und eine Gynäkologin werden zitiert, Lebensschützer_innen, Mitglieder des Parlaments, sogenannte Laien aus Großbritannien und Papua-Neuguinea, Theoretiker_innen und Ethnograph_innen des Gabentauschs, Aristoteles und Stratherns eigene Untersuchungen sozialer Beziehungen in Mount Hagen bevölkern den Text. Dabei geht es der Autorin um die Sichtbarmachung der Bandbreite von Wissen. Indem sie marginalisierte Formen, Vergessenes, alternative Aktivitäten und Relevanzen, z. B. aus dem Bereich des Alltäglichen und von sogenannten Laien in ihre Überlegungen aufnimmt, und vor allem durch ihre Bezugnahme auf die uns fremden und schwer zu verstehenden Welten-Machenden Praktiken und Vorstellungen der Melanesier, will Strathern die Produktivität solcher, zumeist marginalisierter Wissensformen und kulturellen Praxiselementen zeigen, die, so ihre Überzeugung, für die Entwicklung und Entfaltung alternativer diskursiver und kultureller Möglichkeiten in der Gegenwartsgesellschaft bedeutsam sind (ebd., 11).

Es ist ihr Anliegen, diese anderen Motive und alternativen Sprechweisen im Rahmen einer windigenen Anthropologie» (Strathern 1992a, 7) empirisch zu dokumentieren und sie gleichzeitig in die eigene Denk- und 
Begriffsarbeit und das heißt, in die Denk- und Begriffsarbeit euro-amerikanisch-globalisierender Gesellschaften, einzuspeisen. Die niederländische Wissenschaftsforscherin Annemarie Mol bezeichnet diese Form der Analyse als "Inklusion ohne Assimilation«: »Strathern tells about differences that are not exclusive. She draws an image of how a son, contained in, may also contain the father. Of male and female forms that hold the other within. The other, she explains, isn't necessarily elsewhere. It may just as well be incorporated within the self. But it is incorporated without being assimilated« (Mol 2002, 148). Paul Rabinow schreibt im Klappentext des Sammelbands, in dem der hier übersetzte Text erschienen ist, dass Strathern das leiste, was Ethnologie immer behaupte zu tun, aber sehr selten einlöse. Nämlich: Wissen über die eigenen (und eben nicht primär über fremde) Gesellschaften bereit zu stellen.

Wie aber genau nutzt die Autorin das zunächst fremde / andere / marginalisierte Wissen, um das Verstehen der eigenen Gesellschaft voranzutreiben? Wie gelingt es ihr, Vielfalt von Wissen und Kultur produktiv zueinander in Beziehung setzen?

Vielstimmigkeit und Vielfalt von Konzepten und Vorstellungen haben bei Strathern die Funktion, das Denken in den Oppositionen von eigen / fremd oder selbst/anderen zu erweitern; sie werden verwendet, um eine "Anthropology of the otherwise" (Povenelli 2011), des Anderenfalls zu betreiben. Sie erprobt die Tragfähigkeit anderer Modelle und die skeptische Befragung dieser Versuche begleitet und durchzieht ihre Ausführungen, die sie in dem vorliegenden Text als »behindert" und "beschränkt" beschreibt: "Anderes Wissen gleicht nicht notwendigerweise eigene Defizite aus" (Strathern 1992, 120). Situiertheit und Relationalität als methodologische Prinzipien ihres Denkens werden in dem Text quasi vorgeführt und treten als eine besondere Art von Textualisierung und Narrativierung in Erscheinung, in denen unterschiedliche Perspektiven wechselseitig beleuchtet und in Bezug auf das Thema mit- und gegenseitig reflektiert werden; eine relationale Form der Bezugnahme und Integration, die die verschiedenen Welten bewahrt, die in der Perspektiven-Vielfalt stecken.

So ist Stratherns Schreibweise davon bestimmt, die eigene Position des Denkens und Schreibens als relativ, veränderbar, historisierbar, geteilt im vielfachen Sinne in Erscheinung treten zu lassen und die Wechselseitigkeit von Positionalität und Partialität des eigenen forscherischen Tuns sichtbar zu machen. Dazu gehört zentral, wie sie sich in ihrem Schreiben auf Andere bezieht, und deren Wissen, wenn auch immer nur kurz, expliziert und auf diese Weise Relationalität und Situiertheit als Schreibprinzipien vorführt. Hier transportiert sich eine feministische Reflexivität im Sinne 
von Sichtbar-Machung, Wertschätzung und Anerkennung von Erkenntnisbedingungen und -kontexten sowie eine Kritik am westlichen Bild des rautonomen Autors. Denken und Schreiben sind bei Strathern auch auf der Ebene von Textualität relational.

Da Strathern ihren Gegenstand als in Strukturen und Relationen hergestellt denkt und ihn als immer auf die Gesellschaft bezogen und auf sie rückgeführt versteht, ist auch die von ihr verwendete Begrifflichkeit niemals hermetisch. Es gibt in dem Text keine Verabsolutierungen und kein Schreiben im Duktus von Wahrheitsbekundungen. So bleiben die Welten und das Denken offen und es ist nicht immer einfach, die Bedeutung der vorgeführten Relationen zu erfassen; der Text droht der Leser_in immer wieder zu entgleiten.

\section{Feminismus und Geschlecht}

Marilyn Strathern gehörte in den 1970er und 1980er Jahren zu einer Gruppe vornehmlich englischsprachiger Sozial- und Kulturanthropologinnen, die systematisch über Geschlecht nachdachten und sich - in Stratherns Fall auch mit vielen Reibungen - in der feministischen Theorie verorteten. Strathern selbst hat das Verhältnis zwischen Sozial- und Kulturanthropologie und Feminismus einmal als "awkward relationship « beschrieben, als "ein schiefes Verhältnis" (Strathern 1993²), dessen Schieflage unter anderem auch darin begründet sei, dass Geschlecht nicht überall auf der Welt eine Identitäts- und Strukturkategorie ist. In den Gesellschaften Papua-Neuguineas, in denen Strathern als junge Frau gearbeitet hatte, werden Dinge und Personen auf parallele Art und Weise durch den Austausch von Gaben »objektiviert» und in ihrer Besonderheit sichtbar gemacht. Um Reproduktion zu sichern, müssen sowohl Personen als auch Objekte mobilisiert und zirkuliert werden: Nahrungsmittel, Schweine, Muschelschmuck, Kinder. Eine Vorbedingung für den Tausch ist es, dass die beteiligten Personen in der "richtigen Form" sind, sie müssen einen Status von "Unvollständigkeit" annehmen, um Geben, Annehmen und Weiter- oder Zurückgeben zu können (Hirsch 2014, 41). In den Gabentausch-Gesellschaften Papua-Neuguineas sind `Mann und `Frau unvollständige Kategorien; eine Person ist aus sich heraus androgyn, oder crosssex, oder beides. Erst in der Form von `Mann und `Frau können sie Teile ihrer selbst - Wertgegenstände, Verwandte, Tiere - von sich ablösen und

4 Dies ist einer von bisher zwei Texten Stratherns, der auf Deutsch vorliegt. 
geben. Deswegen ist die bedeutsame Differenz in Bezug auf Gender in Mount Hagen, so argumentiert Strathern, nicht eine Differenz von gegebenen Entitäten. Vielmehr verläuft die kulturell bedeutsame Trennung zwischen gleichgeschlechtlichen (männlich-männlich oder weiblich-weiblich) und geschlechtlich-differenten (Mann-Frau-Beziehungen), die wiederum sehr unterschiedliche Formen annehmen können. Strathern beschreibt hier ein relationales und situativ variables Verständnis von Geschlecht, das Geschlecht weder als personal (z. B. `Mann`, ’Frau $)$ noch als gesellschaftlich (z.B. `Geschlechterordnung`) versteht, sondern als eine an Austausch gebundene Praxis und als kulturelle Verhältnisbestimmung.

Dass der Begriff gender/Geschlecht in dem Text »Tauschverhältnisse und Konsumverhältnisse. Der Embryo als zukünftiger Konsument" mit keinem Wort erwähnt wird, ist also nicht einem blinden Fleck in ihrem Denken geschuldet. Vielmehr basiert dieser Aufsatz auf ihrem 400-seitigen Buch "The Gender of the Gift. Problems with Women and Problems with Society in Melanesia« (1988), in dem Strathern ihre Forschungen in Papua-Neuguinea systematisch geschlechtertheoretisch durchdenkt; eine Art opus magnum, das auf die Ausarbeitung eines relationalen Verständnisses von Geschlecht zielt. Angeregt durch die feministische Wissenschaftskritik der 1970er und 80er Jahre unterzieht sie die Forschungen zu melanesischen Kulturen einer umfassenden Reflexion. Sie zeigt, wie Geschlecht in die verschiedenen, soziale Verpflichtungen und Positionen erzeugenden Praktiken des Gebens und Nehmens verquickt ist und als soziale Macht wirkt, und sie nimmt die Situationen des Gabentauschs in Relation zu sozialer Hierarchisierung in diesen Kulturen in den Blick (Strathern 1988, xii). Ihre Erklärungen und Theoretisierungen der klassifizierenden Funktion und der generativ-symbolisierenden Wirkung von gender in Bezug auf die soziale Organisation von Gruppen und Kollektiven, von Räumen und Arealen, von Macht und Arbeit in Melanesien sind durchweg so gearbeitet, dass sie die Unterschiede zu feministischen Ansätzen der Erklärung euro-amerikanischer Geschlechterverhältnisse, Geschlechterbeziehungen und Geschlechterordnungen extrapoliert (ebd., 43-170).

Dabei wird sichtbar, dass anders als in westlichen Kulturen, Menschen in Papua-Neuguinea nicht als geschlechtliche Individuen vorgestellt, sondern in wechselnden Formationen kontrastierender Geschlechtermodi gedacht werden: männlich-weiblich, männlich-männlich, weiblich-weiblich. Darüber hinaus sind diese Beziehungskonstellationen noch darüber differenziert, dass sie direkt (von Person zu Person) oder vermittelt über ein Objekt (z.B. Schwein oder Netztragetasche) konstituiert sind, so dass insgesamt sechs jeweils relational zu anderen Menschen oder Dingen 
praktizierte Formen von Mensch-Umwelt-Konstellationen die Grundlage des melanesischen Selbstverständnisses bilden. So ist Geschlecht zwar auch hier grundlegend-konstitutives Element von Sozialität, es wird aber nicht identitär gefasst, sondern tritt als ein in Austauschbeziehungen hergestelltes, relationales Moment in Erscheinung.

Dieses von Strathern heraus gearbeitete relationale Verständnis von Geschlecht umfasst zwei Perspektiven: eine konzeptionelle (bzw. kategoriale) und eine interaktive. Die erst genannte reflektiert Verbindungen und Relationen, die über eine konzeptuelle Logik und die damit verbundenen Relevanzen hergestellt werden. Die zweite bezieht sich auf Verbindungen zwischen Personen mit ihren jeweiligen Biografien wie auch zwischen Personen und Dingen in ihren spezifischen und situierten Kontexten. Beide Dimensionen gemeinsam in den Blick zu nehmen und gleichermaBen zu beschreiben und zu analysieren, sich zwischen den konzeptionellen Relationen und den interaktiven Bezugnahmen zu bewegen und sie miteinander in Beziehung zu setzen, ist der Kern der kulturanthropologischen Methodik von Strathern (Putnina 2011, 109).

Dementsprechend interessiert sich Strathern weniger für geschlechtliche Klassifikationen von Strukturen und Praktiken im Kontext von Familie, Verwandtschaft, Fortpflanzung und Reproduktion(stechnologien) als vielmehr für das Vermögen der Kategorie Geschlecht, Relationen zu erzeugen und $\mathrm{zu}$ erhalten; unabhängig davon, ob gender nun als Natur oder Kultur, als Biologie oder Soziales, als Gegeben oder Gewählt bestimmt wird (Strathern 2005, 10; Strathern 2016). Für den reproduktionstechnologisch hergestellten Embryo aber, der im Mittelpunkt des hier abgedruckten Textes steht, vermögen Geschlecht und gleichgeschlechtliche oder geschlechtlich-differente Beziehungen (noch?) keine Relationen zu erzeugen und so ist Stratherns relationale Analyse auf die Kategorie ausgerichtet, die diesen thematischen Gegenstand zentral charakterisiert: das Konzept bzw. die Kategorie der >Person

\section{"Extended Remix" als eine etwas andere Form der Gesellschaftskritik - Was bedeutet es, den Embryo als "zukünftigen Konsumenten" zu bezeichnen?}

Thematischer Ausgangspunkt des Textes ist »der Embryo« als Rechtsgegenstand und Objekt parlamentarischer und gesellschaftlicher Debatten. Anders als ein Embryo im Mutterleib verdankt er seine Existenz in dieser Form den Visualisierungs- und Reproduktionstechnologien, die erst er- 
möglichen, dass er als eine isolierte Entität, getrennt von Umwelt, Uterus und Beziehungen in Erscheinung tritt. Strathern bezeichnet diesen Embryo als "zukünftigen Konsumenten" (Strathern 1992, 136), eine Redewendung, die irritiert, vielleicht auch belustigt, und die ungewöhnlich ist, da in den Diskussionen der 1980er-Jahre um Schutz und Personenstatus von Embryonen die Überlegung, dass sich der Embryo zu einem Konsumenten auswachsen werde, keine Erwähnung findet. Warum also stellt Strathern diesen Aspekt heraus und was ist damit gemeint?

Bis 1990 war das Thema Embryonen vor allem auf das werdende Kind im Mutterleib ausgerichtet. Abtreibungsdebatten (z. B. Knecht 1994) wie auch die Kritik an der Medikalisierung von Schwangerschaft (z. B. Duden 1991) reflektierten die Wirkungen und Bedeutung medizinisch-technischer Eingriffe und Apparaturen am Beginn des Lebens im Bauch der Mutter. In den von Strathern betrachteten Konferenzen, Anhörungen und Debatten im Vorfeld der Verabschiedung der ersten Fassung des $\mathrm{Hu}-$ man Fertilisation and Embryology Act 1990 aber tritt der Embryo nunmehr isoliert in Erscheinung, sei es in der Petrischale, in eingefrorener Form im Stickstofftank oder als künstlich befruchtete Eizelle, die in den Uterus eingesetzt werden kann. Diese Sichtbarkeit und Abgetrenntheit Strathern spricht vom Embryo als einer "freistehenden Entität" - trägt mit dazu bei, der Abstraktion eines außerhalb seiner Umwelt stehenden Embryos als »Person« Anerkennung zu verschaffen.

Diese Abstraktion von konkreten Beziehungen und Umwelten kennzeichnet, so arbeitet Strathern in ihrem Text mit Hilfe der Konstrastfolie >Papua-Neuguinea heraus, die westliche Vorstellung von Personalität. $\mathrm{Zu}$ dieser Abstraktion gehört gleichermaßen die Trennung von Öffentlich und Privat sowie die Unterscheidung von Markt vermittelten Beziehungen und vertrauten Beziehungen im Verwandtschafts- und Freundeskreis, »eine Vorstellung von individueller Autonomie (Voluntarismus) und von nach dem Grad ihres Eigennutzes zu bemessenden zwischenmenschlichen Beziehungen (Altruismus) «(Strathern 1990, 20). In westlichen Gesellschaften, so pointiert sie, vergewissern sich "Personen " ihrer selbst und ihrer "Umwelt" durch abstrakte, Markt vermittelte Austauschbeziehungen einerseits und durch Gabentausch im Privaten »als Ausdruck von deren Willen und sozialer Verbundenheit und Verpflichtung« (ebd.) andererseits. Beide Formen der Austauschbeziehungen sind von denselben Prinzipien geleitet, nämlich: Sichtbarkeit (der Güter und Gaben) und Abgetrenntheit (der Einzel-Person); das Geben von Geschenken sei "eine stark personalisierte Form von Transaktion" (ebd., 121) von Gütern und Dingen. Und gleichermaßen halten beide Formen von Austauschbeziehungen Sozialität 
in Gang und dienen der sozialen und gesellschaftlichen Reproduktion im umfassenden Sinne. So gesehen folgen die Spende von Samen, Eizellen und Embryonen und ihre Aneignung als Objekt von Forschung oder als Objekt des Begehrens derselben Logik, nämlich einer, die zwischen `Gaber und 'Ware unterscheidet, die >Person nen als unabhängig von ihren Beziehungen und Umwelten konzipiert.

Dieses Spezifikum westlichen Denkens setzt Strathern in Beziehung zu melanesischen Vorstellungen von >Person raus, dass in Melanesien Aufeinander-Bezogen-Sein als vitale Form der Unterstützung und der Reproduktion aller lebenden Personen und aller signifikanten Dinge praktiziert werden und dass die Differenzierung und Unterscheidung einzelner $>$ Personen $<$ und $\gg$ Dinge $<$ voneinander in aktiver, kultureller Arbeit (z.B. durch den Austausch von Gaben) hergestellt werden müsse. Im Unterschied dazu begriffen Euro-Amerikaner_innen >Personen als basale, beinahe naturhafte Gegebenheit, mit der Folge, dass Beziehungen und Kontakte $\mathrm{zu}$ anderen die Form einer freien, öffentlichen Unternehmung annähmen. In der Perspektive des /Westens` erscheinen umgekehrt dann melanesische Tauschgesellschaften in besonderer Art und Weise durch die Zwänge und Unfreiheiten normativer Reziprozität geprägt.

Stratherns Beschreibung und Analyse unterminiert nicht nur diese zutiefst ethnozentrische Bewertung. Sie schärft vor allem den Blick auf die Charakteristika der westlichen Welt, in der sich reproduktive Prozesse im Kontext der "Konsumkultur» bzw. der "unternehmerischen Gesellschaft» (Strathern 1992a, 8) vollziehen und in welcher Praktiken der Individualisierung und Veränderung der >Person als Konsumentscheidungen realisiert werden. Darüber hinaus ist die Parallelisierung von Euro-Amerika und Melanesien für Strathern auch mit der Frage verbunden, welche Alternativen es zu der Vorstellung von Embryonen als >Personen` gibt und wie Reproduktion jenseits der Konsumkultur aussehen könnte. Oder anders formuliert: Welche Einsichten und Dynamisierungen wären damit verbunden, die mit Hilfe der Reproduktionstechnologie hergestellten Embryonen anders denn als "zukünftige Konsumenten« zu fassen? Um aber über gesellschaftliche Alternativen nachdenken zu können, muss die Wahrnehmungsfähigkeit für Differenz in Geschichte und Gegenwart gestärkt werden. Und zwar Differenzen hinsichtlich aller grundlegenden Wissens-Kategorien: Person, Person-Werdung, Gabe, Beziehung. Dies ist keinesfalls banal - denn auf den ersten Blick können wir für uns Überraschendes, Unbekanntes und Unerwartetes immer nur in den durch unsere Kultur zur Verfügung gestellten Kategorien wahrnehmen. Strathern leis- 
tet mit ihren Texten diese Arbeit, das Vertraute auf überraschende (dezentrierende) Art und Weise zu etwas Unvertrautem zu machen, und das Unvertraute als komplexes Geflecht von ähnlich und fremd, gleich und anders hervortreten zu lassen.

Die von Strathern in ihrem Text vielfach vorgenommenen Analogisierungen und Parallelisierungen sind Versuche, das (Nach)Denken in Gang zu halten und es anzureichern; ihre Formulierung von Entsprechungsverhältnissen zwischen Melanesien und Euro-Amerika sowie die zwischen biologischer Reproduktion, sozial-gesellschaftlicher Reproduktion und kosmologisch/diskursiver Reproduktion sind suchend, erprobend und befragend. Entlang von Vergleichen und Analogisierungen nähert sie sich der Frage nach dem Verhältnis zwischen, erstens, der gesellschaftlichen Reproduktion des Waren-und Konsumkapitalismus, zweitens, der sozialen Reproduktion von Beziehungen zwischen Paaren, Erwachsenen und Kindern oder anderen familialen Gemeinschaften und drittens der biologischen Reproduktion. Durch das Einbringen anderer Formen von Wissen und diverser Stimmen aus unterschiedlichen Gesellschaften, Epochen und Disziplinen in der Form eines "Extended Remix" (Viveiros de Castro/Goldman 2017) entwickelt Strathern verschiedene Lesarten auf das Thema Reproduktion und hält damit den politischen Raum zur Gestaltung des Themenfelds offen.

"Der Embryo als zukünftiger Konsument« ist reproduktionsmedizinisch erzeugt, von Eltern in Auftrag gegeben und von Laborant_innen und Ärzt_innen bearbeitet. Indem er zum Objekt von Arbeit, Tausch und Wert wird, zum Gegenstand einer als altruistisch konzipierten »Gabe« und einer als voluntaristisch imaginierten Reproduktionsentscheidung unterliegt er auch einer veränderten Wahrnehmung. Deren kulturelle Bedeutung (für den Embryo selbst wie für seine Verwandtschaft) ist jedoch noch ungewiss. Und diese Ungewissheit versteht Strathern als Deutungsoffenheit, die es über die dominanten und hegemonialen Konzepte hinausgehend mit Alternativen anzureichern und zu gestalten gilt. Das betrifft die westliche Konzeption von »Person" als autonomem Subjekt, die den Bezug zu den anderen und zur Umwelt vernachlässigt; eine Überlegung, die schon Norbert Elias gegen die Idee des »homo clausus» stark gemacht hat (Elias 1991). Ihre Skepsis und Kritik richtet sich aber gleichermaßen gegen ein faktizistisches, objektivistisches Moment im westlichen Denken und dessen Art von Truism. Und so arbeitet Strathern statt Überzeugungen und Wahrheitspostulaten Entwürfe und tentative Überlegungen aus. Indem sie den "Embryo als zukünftigen Konsumenten« entwirft, fragt sie, ob Welt wirklich so ist, wie wir sie verstehen, ob wir wirklich wollen, dass es im- 
mer so weiter geht und welche anderen Welten vorstellbar und realisierbar sind (Schramm 2017, 471).

Dies ist eine Form von Kritik, die weder Krisen-Rhetorik noch Technik-Verdammung betreibt und die gesellschaftliche Zukunft jenseits von Bio-Ethik und Technikfolgenabschätzung befragt. Anders als die Missbilligung der "wunscherfüllenden Medizin" (Kettner 2000) und im Unterschied zu kapitalismuskritischen Perspektiven auf "Bio-Ökonomie" (Cooper 2000) und der technikphilosophischen Kritik an der Inwertsetzung der Gattung und der Kommerzialisierung der Fortpflanzungsmedizin (Gehring 2016), bildet der Waren- und Tauschcharakter des Embryos und der ihn organisierenden Technologien im Denken von Strathern eher den Ausgangspunkt als den Endpunkt. Sie versteht die mit den Biotechnologien und Reproduktionstechnologien verbundene Ökonomisierung als hegemoniale kulturelle Form, deren Bedeutung für Denken und Verstehen zwar dominant, aber eben nicht total ist. ${ }^{5}$ Deshalb ist es ihr - ähnlich wie Donna Haraway - ein zentrales Anliegen, Denkbewegungen anderer Art und Herkunft in Gang zu halten und sie für das Verstehen der Gegenwart zu nutzen. Während Haraway (2016) sich vor allem auf experimentelle und postmaterialistische Formen bezieht, arbeitet Strathern mit verschiedenen Formen empirisch auffindbaren Wissens, das es ermöglicht, sich zu dem Gegenstand erneut und anders in Beziehung zu setzen. "Studying the relation between the relation" (Lebner 2017, 15) nennt Ashley Lebner Stratherns Arbeitsweise, mit der sie ein Denken entstehen zu lassen bestrebt ist, in dem Relationalität nicht nur als Konzept verwendet wird, sondern eine fortgesetzte lebensweltliche wie auch wissenschaftliche Praxis ist.

Dieses Bemühen um eine relationale und symmetrische Anthropologie, die das Thema `Assistierende Reproduktionstechnologien ‘ durch kritische Befragung und Erweiterung scheinbar gegebener Grundkategorien unseres Denkens und Handelns in erweiterter Form zur Debatte stellt, entwickeln Marilyn Stratherns Kolleginnen und Schülerinnen Sarah Franklin, Jeanette Edwards, Monica Konrad, Charis Thompson und viele andere mit ihren eigenen Forschungen weiter. Ihnen gebührt das Verdienst, dieses Denkprinzip weiter umgesetzt und dafür gesorgt zu haben, dass diese eher implizite Form der Gesellschaftskritik nicht selbst ein Embryo geblieben ist.

5 Dementsprechend spielen auch Ansätze, die zu einer emphatischen und politischen Nutzung von Technologien aufrufen, wie sie beispielsweise Shulamith Firestone (1978) oder auch das Xenofeministische Manifest (2015) entwickelt haben, im Denken von Strathern keine Rolle. 


\section{Fazit}

Stratherns Beschreibung des reproduktionstechnologisch erzeugten Embryos bewegt und verwundert. Bewegung entsteht durch die Verschiebung des Blicks auf ungewöhnliche und abseitige, aber sehr wohl empirisch belegte Denk- und Sichtweisen. Anders als die herkömmlichen gesellschaftlichen Bedenken gegenüber der Verbreitung von Techniken assistierter Reproduktion $-z$. B. Kritik an der Künstlichkeit und einer entgrenzenden Machbarkeit, an neuen Formen von Ungleichheit und Ausbeutung, Angst vor Monstrosität und Befürchtungen gegenüber einer Aneignung der Gebärfähigkeit von Frauen durch eine von Männern dominierte Technik zeigt Strathern, dass sich die konsumgesellschaftlich und neoliberal geprägten Konzepte von Person, Verwandtschaft, Gabe, Reproduktion und Gesellschaft unter dem Einfluss der Reproduktionstechnologien weniger verändern als vielmehr in technologisch angereicherter Form fortschreiben und dabei die gegebenen Kategorien eher noch verfestigen. Und sie stellt kritisch in Frage, inwiefern die vorhandenen Begriffe und Vorstellungen zur Erfassung des technologischen und wissenschaftlichen Wandels, unserem Gebrauch dieses Wandels und den im Zuge dieser beiden Bewegungen entstehenden sozialen Beziehungskonstellationen taugen und was ihre Grenzen sind.

Ihr Fokus liegt also nicht auf den durch die Technologien der assistierten Reproduktion eingebrachten Neuerungen, sondern darauf, dass die diesbezüglichen Entwicklungen und Veränderungen mit Hilfe alter, bekannter Kategorien angeeignet werden. Sie hält die technischen Innovationen für weniger interessant als die kulturelle Kontinuität, welche die Technik hervorgebracht hat und sie strukturiert; die Bezeichnung des Embryos als "zukünftiger Konsument« will genau darauf verweisen. Die reproduktionsmedizinische Herstellung von Leben mag spektakulär und grundlegend erscheinen, solange diese Neuerung aber im Rahmen der Waren- und Konsumkultur gefangen bleibt, bringt sie nichts wirklich Neues hervor. Entgegen gängigen, techno-innovationistischen Vorstellungen eines /Wandels durch Technik ist Strathern davon überzeugt, dass gesellschaftliche Innovation ohne die begriffliche Erfassung der Welt und ohne die Arbeit an Kategorien und Konzepten nicht zu haben ist. Dabei hält sie es für zentral, das im Westen so dominante binäre Denken zu dezentrieren und ihm etwas entgegen zu setzen, Alternativen verfügbar zu halten und diese in das gesellschaftliche Nachdenken einzuspeisen, anderes - relationaleres Wissen sichtbar zu machen und dabei zu helfen, vielfältige Bezüge und Relationen auszuarbeiten. Sorgfältige, manchmal hyperdetaillierte Be- 
schreibungen dienen der Erkennbarkeit und auch Anerkennung komplexer Gleichheits- und Differenzverhältnisse. Strathern erinnert uns daran, dass eine radikale kulturelle und politische Praxis auch darin bestehen kann, das Vertraute auf überraschende Weise zu entfremden, es mit anderen Formen des Weltenmachens partiell zu verbinden und es durch Analogiebildungen zu befragen und zu erweitern. Gemäß der Maxime "Es könnte anders sein« betreibt Strathern Kultur-Analyse im Auftrag des Politischen.

\section{Literatur}

Cooper, Melinda (2008): Life as surplus. Biotechnology and capitalism in the neoliberal era. Seattle.

Duden, Barbara (1991): Der Frauenleib als öffentlicher Ort. Vom Mißbrauch des Begriffs Leben. Hamburg.

Edwards, Jeanette (2000): Born and Bred. Idioms of Kinship and New Reproductive Technologies in England. Oxford.

Elias, Norbert (1991): Die Gesellschaft der Individuen. Frankfurt a. M..

Firestone, Shulamith (1975): Frauenbefreiung und sexuelle Revolution. Frankfurt a. M.

Franklin, Sarah (1993): Making representations: the parliamentary debate on the Human Fertilisation and Embryology Act. In: Edwards, Jeanette / Franklin, Sarah / Hirsch, Eric / Price, Francis/Strathern, Marilyn (Hrsg.): Technologies of procreation: kinship in the age of assisted conception. Manchester, 96-131.

Franklin, Sarah (2002): Embodied Progress: A Cultural Account of Assisted Conception. London: Routledge.

Franklin, Sarah (2013): Biological Relatives: IVF, Stem Cells, and the Future of Kinship. Chapel Hill.

Gehring, Petra (2016): Pille oder Prothese, Pharmakon oder Symbiont. Zwei widerstreitende Fassungen für den menschlichen Technokörper. In: Karin Harrasser, Susanne Rößiger (Hrsg.): Parahuman. Neue Perspektiven auf das Leben mit Technik. Köln, $39-48$

Günther, Peter/Taupitz, Jochen/Kaiser, Hans-Ludwig (2008): Embryonenschutzgesetz. Stuttgart.

Haraway, Donna J. (2016): Unruhig bleiben. Die Verwandtschaft der Arten im Chthuluzän. Frankfurt a.M.

Hirsch, Eric (2014): Melanesian Ethnography and the Comparative Project of Anthropology: Reflection on Strathern's Analogical Approach. In: Theory, Culture \& Society 31 (2/3), 39-64.

Kettner, Matthias (2009): Wunscherfüllende Medizin. Ärztliche Behandlung im Dienst von Selbstverwirklichung und Lebensplanung. Frankfurt a. M.

Knecht, Michi (1994): Zwischen Reproduktionstechnologie und neuer Mütterlichkeit. Verschiebungen und Umbrüche in der Abtreibungsdebatte. In: Wichterich, Christa (Hrsg.): Menschen nach Maß. Bevölkerungspolitik in Süd und Nord. Bonn, 107-128.

Klotz, Maren (2014): (K)information. Gamete Donation and Kinship Knowledge in Germany and Britain. Frankfurt a. M. / New York. 
Konrad, Monica (2005): Nameless Relations: Anonymity, Melanesia and Reproductive Gift Exchange between British Ova Donors and Recipients. New York / Oxford.

Lebner, Ashley (2017): Introduction. Strathern's Redescription of Anthropology. In: dies. (Hrsg): Redescribing Relations. Strathernian Conversations on Ethnography, Knowledge and Politics. New York/Oxford, 1-38.

Mol, Annemarie (2000): The body multiple. Ontology in medical practice. Durham.

Putnina, Aivita (2011): Invisible families: imagining relations in families based on samesex partnership. In: Edwards, Jeanette/Petrovic-Steger, Maja (Hrsg.): Recasting Anthropological Knowledge. Inspiration and Social Sciences. Cambridge, 106-124.

Povinelli, Elizabeth A. (2011): Routes / Worlds. In: e-flux, no. 27.

Schramm, Katharina (2017): Einführung: Postkoloniale STS. In: Bauer, Susanne/Heinemann, Torsten/Lemke, Thomas (Hrsg.): Science and Technology Studies. Klassische Positionen und aktuelle Perspektiven. Frankfurt a. M., 471-494.

Strathern, Marilyn (1981): Kinship at the Core. An Anthropology of Elmdon, a Village in North-West Essex in the Nineteen-Sixties. Cambridge u.a.

Strathern, Marilyn (1988): The Gender of the Gift. Problems with Women and Problems with Society in Melanesia. Berkeley/Los Angeles / London.

Strathern, Marilyn (1991): "Partners and Consumers. Making Relations Visible«. In: New Literary History. A Journal of Theory and Interpretation, Bd. 22 (3), Special Issue "Undermining the Subject«, ed. by Ralph Cohen, 581-601.

Strathern, Marilyn (1992): Partners and Consumers. In: Dies.: Reproducing the Future. Essays on anthropology, kinship and the new reproductive technologies. New York, 118-138.

Strathern, Marilyn (1992a): Introduction: Artificial Life. In: Dies.: Reproducing the Future. Essays on anthropology, kinship and the new reproductive technologies. New York, 1-13.

Strathern, Marilyn (1993): Ein schiefes Verhältnis: Der Fall Feminismus und Anthropologie. In: Rippl, Gabriele (Hrsg.): Unbeschreiblich weiblich. Texte zur feministischen Anthropologie. Frankfurt a. M., 174-195.

Strathern, Marilyn (1997): Partners and Consumers: Making Relations Visible. In: Schrift, Alan D. (Hrsg.): The Logic of the Gift. Toward an Ethic of Generosity. New York/ London, 292-311.

Strathern, Marilyn (2005): Kinship, Law and the unexpected. Relatives are always a surprise. Cambridge.

Strathern, Marilyn (2016): Before and After Gender. Sexual Mythologies of Everyday Life. Edited and with an introduction by Sarah Franklin. Afterword by Judith Butler. Chicago: HAU books.

Thatcher, Margret (1987): Interview. In: Woman's Own 31, 23. September 1987, 8-10.

Thompson, Charis (2007): Making Parents. The ontological choreography of reproductive Technologies. New York.

Viveiros de Castro, Eduardo / Goldman, Marcio (2017): Slow Motions (Extended Remix): Comments on a few texts by Marilyn Strathern. In: Lebner, Ashley (Hrsg.): Redescribing Relations. Strathernian Conversations on Ethnography, Knowledge and Politics. New York/Oxford, 173-196.

Xenofeministisches Manifest (2015): http://laboriacuboniks.net/de (zuletzt abgerufen am 27.8.2018). 